\title{
Synchronization of Wave Flows of Arterial and Venous Blood and Phases of the Cardiac Cycle with the Structure of the Peripheral Pulse Wave in Norm: Part 2
}

\author{
Alexander G. Kruglov, PhD, ScD*; Valery N. Utkin; Alexander Yu. Vasilyev, PhD, ScD; \\ Andrey A. Kruglov, PhD
}

Central Research Institute of Radiation Diagnosis

Moscow, the Russian Federation

\begin{abstract}
Hemodynamic indices studied in practically healthy people were obtained by catheterization in various vascular areas: the chambers of the heart (ventricles, atria, coronary sinus), pulmonary trunk, aorta, inferior vena cava, superior vena cava, right hepatic vein, and sigmoid sinus. Using the mean values of the hemodynamic parameters, we constructed graphics of the "curves" of the central, arterial, and venous pressure, synchronized with each other and an ECG, and with the radial pulse wave recorded by a non-invasive method. The obtained data, which demonstrate the projection coincidences of the characteristic points of peripheral pulse wave with the key indicators of the phases of cardiac cycle, made it possible to transform the results obtained during the invasive examination into indicators of the non-invasive technique. This transformation became possible not only at the characteristic points of the deployed peripheral pulse wave, but in each anacrotic and dicrotic segment, which are understood as the projection areas of the synchronized hemodynamic and wave processes of the vascular bed. We believe it possible to catalog the forms of pulse waves, as well as their projection segments, to obtain accurate diagnostic information about the phases of cardiac cycle and organ hemodynamics in humans in norm and with pathological conditions, using a non-invasive methood based on basic information obtained by invasive methods. (International Journal of Biomedicine. 2018;8(3):177-181.)
\end{abstract}

Key Words: cardiac cycle $\bullet$ hemodynamic parameters $\bullet$ ECG $\bullet$ peripheral pulse wave

\section{Abbreviations}

Ao, aorta; AV, aortic valve; CC, cardiac cycle; CFB, central fibrous body; CS, coronary sinus; EDP, end-diastolic pressure; IJV, internal jugular vein; IVC, inferior vena cava; LA, left atrium; LV, left ventricle; MV, mitral valve; PT, pulmonary trunk; PPW, peripheral pulse wave; PW, pulse wave; PV, pulmonary valve; RV, right ventricle; RA, right atrium; RHV, right hepatic vein; SS, sigmoid sinus; SVC, superior vena cava; TV, tricuspid valve; ZTEP, zone of temporal equalization of pressure.

\section{Basic Part}

The aim of this study was to compare and extrapolate the results of invasive and non-invasive diagnostic techniques in humans under normal and pathological conditions.

*Corresponding author: Alexander G. Kruglov, PhD, ScD. Central Research Institute of Radiation Diagnosis. Moscow, the Russian Federation.E-mail: krag48@mail.ru

\section{Methods and Discussion}

The parameters of the hemodynamics of the central, arterial, and venous blood flow have been discussed in detail and repeatedly published. ${ }^{(1-3)}$ Based on the mean values of hemodynamic parameters obtained by catheterization - in the heart (RV, LV, RA, LA, CS), Ao, PT, liver, kidney and SS - we constructed graphs of the sequential dynamic pressure change in the each studied point, which were synchronized with each other, ECG and with a detailed single PPW. ${ }^{(4)}$ Synchronized 
wave flows and PPW are shown in Table 1. Numbers and colors indicate PW (blue color) and the vessels and organs in which the catheterization was performed. In the right part (the second (C), the yellow color indicates the graphic sequence of the hemodynamic curves revealed during synchronization: ZTEPs. Separately, the list of projective coincidences of the $\mathrm{CC}$ phases and characteristic points of PW (10 points) is presented.

A partial discussion of the diagram of the sequences of the CC phases presented in Table 1, was given in our previous work. ${ }^{(5)}$ Yellow color indicates a curve that combines the sequence of ZTEPs during CC. Recall that we understand ZTEPs (zones of intersection of hemodynamic curves) as brief (23 points of ZTEPs, marked with Roman numerals, over one CC) periods of zeroing hemodynamic gradients in the investigated zones - periods of equilibrium states of both consecutive and distributed parts of the vascular system.

We believe that ZTEPs that have a fixed sequence (indicated on the graph) form a high-speed matrix of control hemodynamic points, which ensure the sequence (phasing) and synchronism of the phase periods of CC (considering peripheral ZTEPs, possibly regulation of the vascular system as a whole). We consider it necessary to note the exact topographic coincidence of a significant part of the peak ZTEP values with all the characteristic points of the detailed peripheral pulse curve: 1-10 points (marked by vertical lines, Table 1).

The mismatch (delay) of the peak values of the arterial part of the hemodynamic curves is due to the outrunning spread of the wave impulse (PW) along the vascular bed in comparison with the speed of distribution of the arterial blood substrate after the systole of LV.

Recall that an impulse of PW reaches all metabolic zones of the body in the interval from the opening of AV to closing of $\mathrm{AV} .{ }^{(6)}$ We believe (intentionally simplifying the structure of connections of hemodynamic flows) that the centrifugal controlling informational impulse (PW), having passed the exchange zones of organs (i.e., the mosaic peripheral resistance, which depends on the activity of exchange and intensity of local blood flow), is transformed into a network of afferent information flows of feedback with the concentration in RA to prepare the next CC.

Modern methods of non-invasive examination of PW do not have a direct appeal to heart hemodynamics guided by indirect signs (including oscillations and vibrations of the chest of various genesis) of the heart functioning. We studied these methods across the whole range of available information, regardless of the method and localization of the initial information signal, and quantitatively assessed the characteristics of PW, followed by differentiation of its species, cataloging, and expert diagnostic evaluation, One of the most accurate methods is to measure the phases of $\mathrm{CC}$ according to a high-speed kinetocardiogram $(\mathrm{KCG})^{(4)}$ synchronous with the reference curves: ECG, phonocardiogram, and sphygmogram of the carotid artery. KCG indirectly measures the following time intervals: the duration of $\mathrm{CC}$ (R-R), the phase of asynchronous contraction, the phase of isometric contraction, the phase of rapid ejection, the phase of slow ejection, and the systole of the atrium, without the ability to accurately measure the inside and interphase dynamics or connections with organ hemodynamics.

Most researchers believe that the basic information about the state of health is represented by the elements of a single PW of the radial artery that has a stable character. The main informational elements are the amplitude-time parameters determined by the shape (contour) of the pulsogram (local maxima-minima inflection points). A high degree of similarity in the contour of the pulsograms of the carotid and radial arteries was established, which allowed to transfer the methods of not only contour analysis, but also of the phase analysis from the curves measured in the heart and the aortic aperture to the pulsogram of the radial artery. ${ }^{(7)}$ As a mathematical apparatus, a contour pulsogram analysis was used by the methods of spline approximation and regularization. The most variable regions of PW by analyzing the statistical nature of the dynamic series of amplitude-time parameters of the characteristic points of long realizations of PW are identified. This variant of mathematical processing made it possible to use the method for contour analysis of a pulsogram (sphygmogram) as an effective analysis of biomedical information.

The method is based on detecting amplitude-time parameters in 10 informative points of $\mathrm{PW}$, characterizing not only the phases of CC ( 9 temporal intervals corresponding to projections on time axis), but also the shape of PW. Point 10 (the end of PW) is simultaneously the starting point (Point 1) of the next wave. It was also found that the distribution of the amplitudes of the dynamic series of the pulse does not correspond to the known distribution laws of random variables. $^{(4,7,8)}$

The results we obtained during the catheterization are synchronized with the PW graph (radial artery) that was obtained by a non-invasive method, with a single cycle of PPW having allotted characteristic points. Ten characteristic points of PW, which determine the primary amplitude-time parameters, have highly accurate characteristics due to use of effective mathematical algorithms. ${ }^{(8-10)}$ This approach made it possible to determine reliable projection coincidences (Table 1) between characteristic points of PPW and the phases of CC.

The revealed projective coincidences allow us, at the initial stage, to produce an accurate marking of PPW according to the phases of $\mathrm{CC}$ and organ hemodynamics, obtaining reliable information on the hemodynamic parameters by a non-invasive way. The results of synchronization of central hemodynamics and PPW (radial artery - characteristic points from 1 to 10) revealed the presence of the following projection coincidences of the characteristic points of $\mathrm{PW}$ with the phases of CC: Point 1: a coincidence with the closing of MV; Point 2: with ZTEP for LA (med) - SS (min); Point 3: with ZTEP for CS (maximum) and SS (maximum); Point 4: with the opening of AV; Point 5: with the closing of PV; Point 6: with the opening of TV; Point 7: with the closing of AV; Point 8: with the opening of MV; Point 9: with ZTEP for LA and LV (diastole); Point 10: with the closing of MV.

Thus, the possibility of non-invasively extracting information on the factual state of cardiovascular hemodynamics in general is presented. 


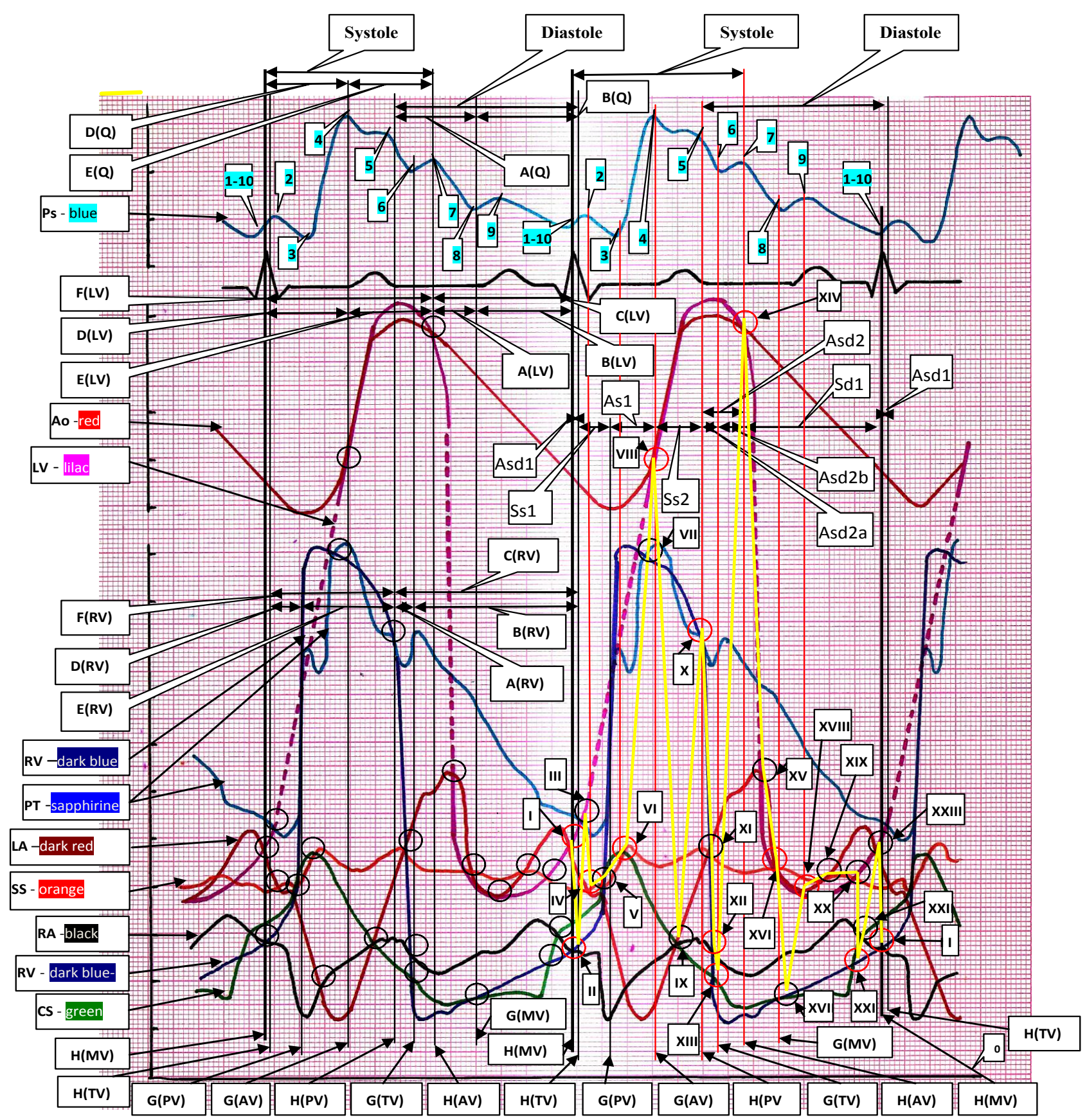

\begin{tabular}{|c|c|}
\hline \multicolumn{2}{|c|}{ Table 1. Coincidences between characteristic points of PPW and the phases of CC in Norm } \\
\hline $\begin{array}{l}\text { Coincidences of the characteristic points of PW (V.V. Boronoev) with the phases of CC: } \\
\text { 1 - with the closing of MV } \\
\text { 2- with ZTEP for LA (med) - SS (min) } \\
\text { 3 - with ZTEP for CS (maximum) and SS (maximum) } \\
\text { 4- with the opening of AV } \\
\text { 5 - with the closing of PV } \\
\text { 6- with the opening of TV } \\
\text { 7 - with the closing of AV } \\
\text { 8 - with the opening of MV } \\
9 \text { - with ZTEP for LA and LV (diastole) } \\
10(1) \text { - with the closing of MV } \\
\text { ro - Characteristic points of PW (V.V. Boronoev) } \\
\text { A(Q) - isometric ventricular relaxation } \\
\text { B(Q) - actual ventricular diastole } \\
\text { C(LV) - LV diastole } \\
\text { A(LV) - isometric relaxation of LV } \\
\text { B(LV) - actual LV diastole } \\
\text { C(RV) - RV diastole } \\
\text { A(RV) - isometric relaxation of RV } \\
\text { B(RV) - actual RV diastole } \\
\text { D(Q) - isometric ventricular contraction } \\
\text { E(Q) - actual ventricular systole } \\
\text { F(LV) - LV systole }\end{array}$ & $\begin{array}{l}\text { E(LV) - actual ventricular systole } \\
\text { F(RV) - RV systole } \\
\text { D(RV) - RV isometric contraction period } \\
\text { E(RV) - actual RV systole } \\
\text { G(AV) - opening of AV } \\
\text { H(PV) - closing of PV } \\
\text { G(TV) - opening of TV } \\
\text { H(AV) - closing of AV } \\
\text { G(MV) - opening of MV } \\
\text { H(TV) - closing of TV } \\
\text { H(MV) - closing of MV } \\
\text { G(PV) - opening of PV } \\
\text { H(PV) - closing of TV } \\
\text { Asd1 - asynchronous period of ventricular systole-diastole -1 } \\
\text { Ss1 - synchronization period of isometric ventricular contraction-1 } \\
\text { As1 - asynchronous period of ventricular systole -1 } \\
\text { Ss2 - synchronization of the actual ventricular systole -2 } \\
\text { Asd2 - asynchronous period of ventricular systole-diastole -2 } \\
\text { Asd2a - from the closing of PV to the opening of TV } \\
\text { Asd2b - from the opening of TV to the closing of AV } \\
\text { Ad1 - asynchronous period of ventricular diastole -1 } \\
\text { Sd1 - period of synchronization of ventricular diastole-1 } \\
\\
\end{array}$ \\
\hline
\end{tabular}




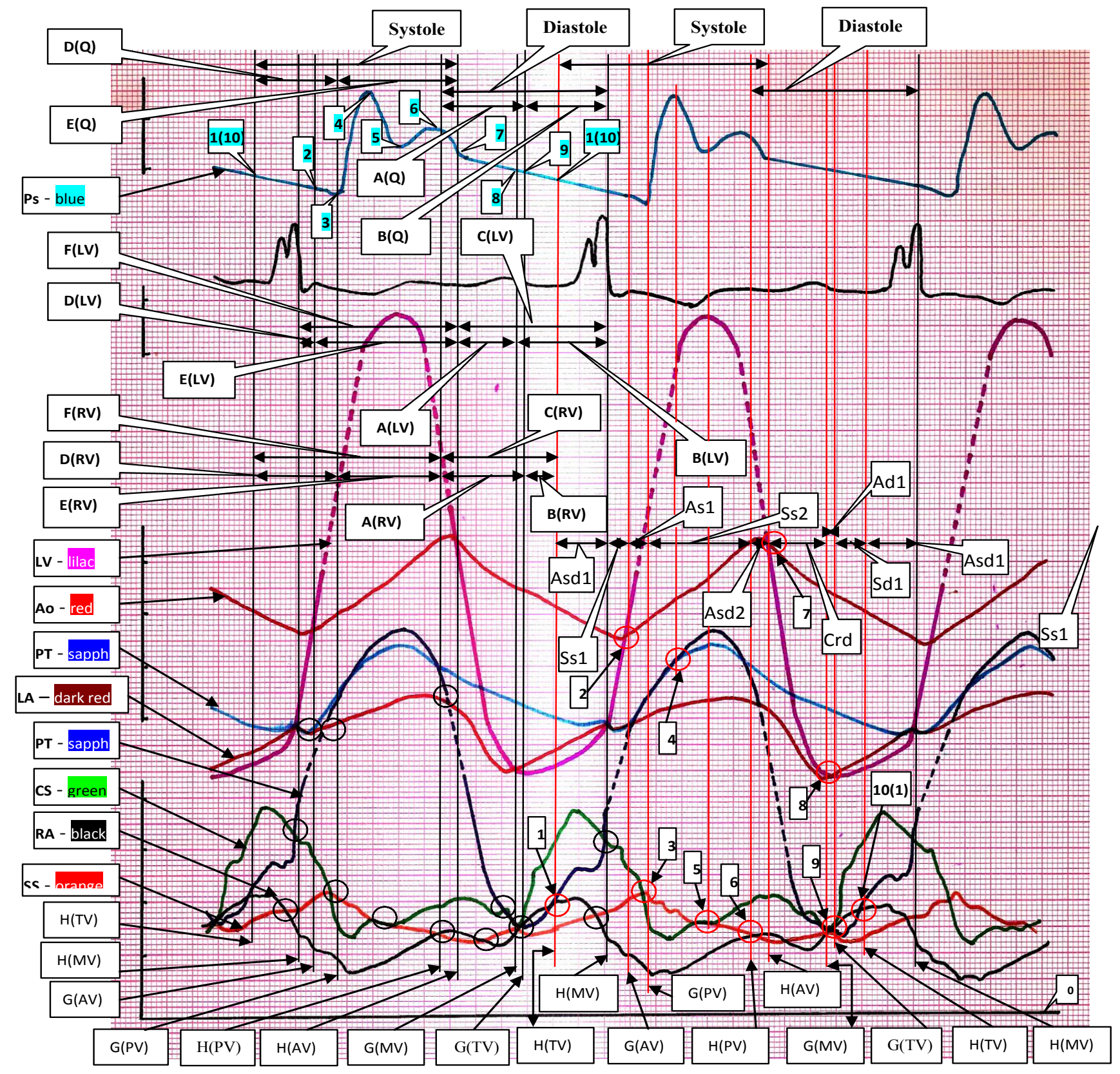

\begin{tabular}{|c|c|}
\hline $\begin{array}{l}\text { Coincidences of the characteristic points of PW (V.V. Boronoev) with the phases of CC: } \\
\text { 1 - with the closing of TV } \\
\text { 2- with the opening of AV } \\
\text { 3 - with the opening of PV } \\
\text { 4 - with ZTEP for PT and RV } \\
\text { 5 - with ZTEP for CS (minimum) and SS (middle) } \\
\text { 6 - with the closing of PV } \\
\text { 7- with the closing of AV } \\
\text { 8 - with the opening of MV } \\
\text { 9- with ZTEP for RA, CS, PT, SS } \\
\mathbf{1 0 ( 1 )} \text { - with the closing of TV } \\
\text { - Characteristic points of PW (V.V. Boronoev) } \\
\text { A(Q) - isometric ventricular relaxation } \\
\text { B(Q) - actual ventricular diastole } \\
\text { C(LV) - LV diastole } \\
\text { A(LV) - isometric relaxation of LV } \\
\text { B(LV) - actual LV diastole } \\
\text { C(RV) - RV diastole } \\
\text { A(RV) - isometric relaxation of RV } \\
\text { B(RV) - actual RV diastole } \\
\text { D(Q) - isometric ventricular contraction } \\
\text { E(Q) - actual ventricular systole } \\
\text { F(LV) - LV systole }\end{array}$ & $\begin{array}{l}\text { D(LV) - LV isometric contraction period } \\
\text { E(LV) - actual LV systole } \\
\text { F(RV) - RV systole } \\
\text { D(RV) - RV isometric contraction period } \\
\text { E(RV) - actual RV systole } \\
\text { G(AV) - opening of AV } \\
\text { H(PV) - closing of PV } \\
\text { G(TV) - opening of TV } \\
\text { H(AV) - closing of AV } \\
\text { G(MV) - opening of MV } \\
\text { H(TV) - closing of TV } \\
\text { H(MV) - closing of MV } \\
\text { G(PV) - opening of PV } \\
\text { Asd1 - asynchronous period of ventricular systole-diastole -1 } \\
\text { Ss1 - synchronization period of isometric ventricular contraction -1 } \\
\text { As1 - asynchronous period of ventricular systole -1 } \\
\text { Ss2 - synchronization of the actual ventricular systole -2 } \\
\text { Asd2 - asynchronous period of ventricular systole-diastole -2 } \\
\text { Crd- asynchronous period of isometric relaxation of the ventricles } \\
\text { Ad1 - asynchronous period of ventricular diastole -1 } \\
\text { Cd1 - period of synchronization of ventricular diastole-1 } \\
\text { - - coincidence of invasive and non-invasive points (V.V.Boronoev) }\end{array}$ \\
\hline
\end{tabular}


Taking into account the stability of a single PW, we believe it is possible and expedient to carry out accurate projection marking of the whole set of synchronized hemodynamic curves throughout the entire graph of PPW. Full marking with precise binding of each segment of PW to the stages of specific wave and hemodynamic processes of the vascular bed as a whole will allow determining their correspondence at any time, using a non-invasive method of access. Detailed segmental marking of PW by the proposed method will allow creating an address cataloging the shapes and segments of PW for the norm (including under different loading regimes) and various types of clinical pathology based on objective data obtained by invasive methods. We believe that the key signs of targeted diagnostic interpretation will be, in addition to variations in the shape of PW itself, changes in the shape, duration, angularity and other characteristics of the corresponding segments of the pulse curve, which are the projection mapping of the hemodynamics of a particular vascular or organ process.

A similar study by catheterization was conducted in the group of patients $(n=247)$ with acquired heart defects. Below are given the hemodynamic data of patient S., a 47-year-old man with a mixed aortic valve disease with prevalence of aortic valve stenosis, and stage 2 pulmonary hypertension (Table 2 ).

The defeat of the valvular apparatus of the heart with acquired defects leads to a loss of the sealing of the chambers of the heart in the phase of isometric contraction of the ventricles due to a disturbance in the closing ability of the valves, in combination with excessive pressure in the heart chambers (stenosis, insufficiency). The consequence is a disturbance in the controlling waveforms generated by the myocardium, as well as distortion of the temporal relationships of the phases and periods of CC (including changes in the topology and composition of the "pressure equalization zones"), leading to an imbalance and desynchronization of the regulation of hemodynamic and wave processes, both in the chambers of the heart .

Note the change in the shape of PW and the radical differences between the projection coincidences of the phases of CC and PPW (Table 2) at the characteristic points: Point 1: with the closing of TV; Point 2: with the opening of AV; Pont 3: with the opening of PV; Point 4: with ZTEP for PT and RV; Point 5: with ZTEP for CS (minimum) and SS (middle), Point 6: with the closing of PV; Pont 7: with the closing of AV; Point 8: with the opening of MV; Point 9: with ZTEP for RA, CS, PT, SS; Point 10: with the closing of TV.

A radical difference between the norm (Table 1) and aortic valve disease (Table 2) in the whole spectrum of coincidences of the key indexes of the CC phases with the characteristic points and projection segments of PPW will be examined in detail in the further works.

\section{In conclusion:}

Synchronizing the graphic curves of the central, arterial, and venous pressure that were obtained by an invasive method during catheterization with ECG and a detailed single-pulse curve obtained by a non-invasive method on the radial artery, revealed the projection coincidence of the key points of PW with the sequence of key phases of $\mathrm{CC}$ in the norm.

The coincidence of the key points of PPW, considered as reference points, directly with the key points of cardiovascular hemodynamics and the peak values of ZTEPs, enables the targeted marking of the entire length of the radial pulse wave, where each anacrotic/dicrotic segment is considered as a projection element of the wave and hemodynamic processes of the vascular bed as a whole, both in the norm and under pathological conditions.

The exact attachment of each segment of PPW to a specific fragment of the wave/hemodynamic process of the vascular bed allows determining their relationships and correspondences in any time interval using non-invasive access methods, where the diagnostic set, in addition to PW itself, is the shape, duration, angularity and other characteristics of the corresponding segments of PW, which are the projection mapping of the hemodynamics of a particular vascular or organ process.

\section{Competing interests}

The authors declare that they have no competing interests.

\section{References}

1. Gebel GYa, Kruglov AG, Utkin VN, Bagdatyev VE, Dasaev AN, Golostenova LM. [On the role of the coronary sinus of the human heart in the norm (regulation of a number of functions to the issue of synchronization in the circulatory system)]. Proceedings of the 10th Conference on Space Biology and Aerospace Medicine. M., 1994:56-57. [Article in Russian].

2. Kruglov AG, Vasilyev AY, Sherman VA. Human dynamic homeostasis control matrix in the norm with psychophysiological aspects. New-York: IMRDC; 2016.

3. Kruglov AG, Utkin VN, Vasilyev AY, Sherman VA. Human Homeostatic Control Matrix in Norm. International Journal of Biomedicine. 2016;6(3):184-9.

4. Boronoev VV. [Pulse wave contour analysis in automated mode].Med Tekh. 2014;(4):33-6.[Article in Russian].

5. Kruglov AG, Utkin VN, Vasilyev AY. Synchronization of Wave Flows of Arterial and Venous Blood with Phases of the Cardiac Cycle in Norm: Part 1. International Journal of Biomedicine. 2018;8(2):123-128.

6. Lightfoot A. Transport phenomena in live systems. Biomedical aspects of momentum and mass transport. M.: Mir; 1977. [In Russian]

7. Boronoev VV. Physical basis of pulse diagnostics. Abstract of ScD Thesis. Ulan-Ude; 1999. [In Russian].

8. Boronoev VV, Rinchin OS. Method of Spline Approximation in the Problem of Amplitude-Time Analysis of Pulse Wave. Radiophysics and Quantum Electronics. 1998;4(8):706-15.

9. Boronoev VV, Shabanova EV. [Numerical differentiation of the sphygmogram of the radial artery by A.N. Tikhonov regularization method]. Izmeritelnaya Tekhnika. 1994;(11):6062. [Article in Russian].

10. Boronoev VV. [Practical implementation of pulse diagnosis by instrumental techniques]. Mezhdunarodnyi zhurnal prikladnykh I fundamentalnykh issledovanii. 2015;(12-1):188-192. [Article in Russian]. 